\title{
Demyelination and neurodegenerative changes inserum IgG antibodies to GM1, GD1a and GM3 gangliosides in a patient on dialysis
}

\author{
Vera Kolyovska ${ }^{1 *}$, Velichka Pavlova ${ }^{1}$, Ivan Iliev $^{1}$ and Sonya Ivanova ${ }^{2}$ \\ ${ }^{1}$ Department of Experimental Morphology, Institute of Experimental Morphology, Pathology and Anthropology with Museum, Bulgarian Academy of Sciences, \\ Sofia, Bulgaria \\ ${ }^{2}$ Multiprofile Hospital for Active Treatment in Neurology and Psychiatry “St. Naum”, Sofia, Bulgaria, 1, "Louben Roussev” Street, Bulgaria
}

\begin{abstract}
During the last two decades, an enormous effort has been made to discover biological markers of neuronal damage to effective response to therapy. Gangliosides a family of acidic glycosphingolipids are highly represented in the nervous system. Their spectra show considerable changes in pathological conditions. The complex of anti-ganglioside antibodies may be useful diagnostic and prognostic tool of markers for demyelination (GM1), neurodegeneration (GD1a)and(GM3) correlates with the loss of integrity of the blood brain barrier (BBB). Additionally, patients with renal failure may manifest a variety of neurologic disorders.Dialysis itself is associated with at least three distinct disorders of the central nervous system, including the dialysis disequilibrium syndrome, dialysis dementia, and progressive intellectual dysfunction. Our case studyinvolves a patient on dialysis, a 72-years old woman, as an example of long-term toxicity. She was on dialysis for 1,5 years. The values of IgG anti-GM1, anti-GD1a and anti-GM3 antibodies titers in the blood serum were detected by ELISA. Significantly elevated serum IgG anti-GM1 antibodies titers were detected in our patient. The value of the titer of IgG antibodies against GM1 showed the presence of weak demyelination, increasing with dialysis treatment but not neurodegeneration. On the other hand, other biomarkers were indicative of the preserved integrity of the BBB since IgG titers of antiGD1a and anti-GM3 antibodies were in norm. The patient had many signs that show and prove symptoms of encephalopathy.Neurologic findings in the patients with uremic encephalopathy (UE) range from normal to a comatose state. Neurologic diseases which are specifically related to uremia and/or dialysis occur with increased frequency in patients with end-stage renal disease on chronic hemodialysis. These include subdural hematoma, electrolyte disorders, vitamin deficiencies, drug intoxication, hypertensive encephalopathy, and acute trace element intoxication. Our observations show that there is a relationship between kidney problems and central nervous system (CNS).
\end{abstract}

\section{Introduction}

Patients with renal failure may manifest a variety of neurologic disorders.Unfortunately, despite the adequate maintenance dialysis therapy, some patientscontinue to be afflicted with more subtle nervous dysfunction, including impaired mentation, generalized weakness, and peripheral neuropathy. These central nervous system disorders are referred to as uremic encephalopathy. The dialytic treatment of end-stage renal disease has itself been associated with the emergence of several new disorders of the central nervous system: dialysis dysequilibrium, dialysis dementia and progressive intellectual dysfunction. The dialysis disequilibrium syndrome consists of headache, nausea, muscle cramps, obtundation, and seizures, and is a consequence of the initiation of dialysis therapy in some patients. Dialysis dementia is a progressive, generally fatal encephalopathy which affects patients on chronic hemodialysis [1]. In addition to the foregoing neurologic diseases which are specifically related to uremia and/or dialysis, a number of other neurologic disorders occur with increased frequency in patients with end-stage renal disease on chronic hemodialysis. These include subdural hematoma, electrolyte disorders, vitamin deficiencies, drug intoxication, hypertensive encephalopathy, and acute trace element intoxication [1-3].

During the last two decades, an enormous effort has been made to discover biological markers of neuronal damage, capable to predict disease course and effective response to therapy [4]. Gangliosides are a family of acidic glycosphingolipids highly concentrated in the nervous system, where they represent about $10 \%$ of the total lipid content. Gangliosides are found mainly in the neurons but also occur in smaller concentration in other cell types. The ganglioside spectra of normal blood plasma are remarkably stable, but show pronounced changes in pathological conditions [5]. The main gangliosides in the human central nervous system myelin are monosialogangliosides GM1 [6]. GD1a is one of major central nervous system (CNS) neuronal ganglioside fraction. In our previous studies a considerable increase of serum GD1a ganglioside was determined in multiple sclerosis (MS) - neurodegenerative multifactor disorder with an autoimmune component. Antibodies to gangliosides have been detected in the sera of MS patients, where high titers of IgG anti-GM1 antibodies were

Correspondence to: Vera Kolyovska, Assistant professor, Institute of Experimental Morphology, Pathology and Anthropology with Museum, Department of Experimental Morphology; Address: Bulgaria, Sofia 1113, Acad. G. Bonchev Str., Bl. 25, Tel: + 3592979 2397, E-mail: verakol@abv.bg

Key words: demyelination, chronic kidney disease, dialysis, ELISA, serum IgG antiGM1, anti-GD1aand anti-GM3antibodies

Received: February 05, 2017; Accepted: March 04, 2017; Published: March 06 2017 
associated with demyelination, whereas high titer of IgG anti-GDla antibodies were associated with neurodegeneration, respectively [6]. Concomitantly, high titer of serum IgG anti-GM3 antibodies correlates with the loss of integrity of the BBB. The finding of antiganglioside antibodies in inflammatory demyelination of the CNS may identify avenues for research into pathogenesis.

Physical findings are variable and depend on the severity of the encephalopathy. Neurologic findings range from normal to a comatose state. Cases of Wernicke syndrome associated with uremic encephalopathy(UE) have been described in theliterature, and Wernicke syndrome has been observed in patients with UE, dialysis dementia, or dialysis disequilibrium syndrome.Acute renal injury has been found in mice with increased neuronal pyknosis and microgliosis in the brain. Acute renal injury also led to increased levels of the proinflammatory chemokines keratinocyte-derived chemoattractant and G-CSF in the cerebral cortex and hippocampus, as well as increased expression of glial fibrillary acidic protein in astrocytes. Acute renal injury led to both soluble and cellular inflammation in the brain, affecting the CA1 region of the hippocampus foremost. Acute renal injury leads to increase in brain microvascular leakage [7].

Apart from this, there are suggestions that the number of patients with MS correlates with environmental pollution and an appropriate recommendation to patients is to drink purified water and be careful with their diet [8]. MS is a disease of the polluted environment and cities. Some time ago we conducted an experiment in animals, which proves intoxication affects glial cells.

\section{Materials and methods}

In our study a 72-years-old female patient fulfilled all diagnostic criteria for clinically definitekidney failure. She had a history of episodes of longitudinalkidney failureand one functioning kidney. Her exacerbations were treated three times per week by hemodialysis. During the blood sampling the patient was in good general physical condition, took food, with variable appetite and changeable vitality, but the mental condition of the patient was not normal. Her blood tests were done regularly at Dialysis department of UMHATEM "N. I. Pirogov", Sofia and were in normal range.The blood, for our tests, had no need for centrifugation, due to the addition of anticoagulants required for dialysis. Six months after our study patient died.

The presence of the encephalopathy can lead to slow changes over time, changes that are not easy to diagnose. Despite numerous and varied causes of encephalopathy one of the symptoms present in all encephalopathy cases and these are changes in mental state. It can develop slowly or be obvious or develop rapidly and lead to coma or death. Often, symptoms of altered mental status may be inattentiveness, poor judgment or poor coordination of movements.

Other serious symptoms which may occur with encephalopathy are lethargy, dementia, seizures, tremor, muscle spasm and myalgia, changing patient's breathing pattern observed with brain damage and coma. In one way or another, our patient had all these symptoms. Often, the severity and type of symptoms are related to the severity and cause of the disease or brain damage. The sick had increasingly poor memory for recent events and activity of thinking was delayed. All these signs are symptoms of encephalopathy.

She had a spastic gait with inversion on the right foot with difficulty in speaking, inadequate. The patient was disoriented and inattentive; both recent and remote memory was impaired. Speech was distorted and was marked by frequent arrests. Both lower limbs were weak, right, slightly more than the left. The syndrome of chronic dialysis encephalopathy is fairly consistent as a clinical picture. In the majority of patients, it is a disorder of speech and language features camping, hesitant dysarthria frequent arrests of speech literal and phonemic paraphasias and relatively preserved comprehension. Disorder of speech may vary depending on the schedule of dialysis; deterioration during and after dialysis. Multifocal myoclonic movements occur involving the extremities and face, with seizures appear later in the course of the disease. Various psychiatric symptoms can occur, all against the backdrop of progressive dementia. So our patient did indeed have an encephalopathy.

Electromyography (EMG) was also used to exclude demyelination polyneuropathy of the peripheral nervous system (PNS). All the above signs are characteristic for MS patients and our experience with MS patients and demyelination and neurodegeneration data bowed down us to think for the such processes.Our scientific curiosity led us to include her serum to the plates with many of our patients with a confirmed diagnosis of MS and to interpret the results.

\section{ELISA Protocol}

The serum anti-GM1, anti-GD1a and anti-GM3 antibodies were estimated by the enzyme-linked immunosorbent assay (ELISA) with some slight modifications of the method used by Ravindranath [9]. Briefly, thousand nanograms of GM1 (or GD1a, or GM3) ganglioside (Sigma) in $100 \mathrm{ml}$ of methanol were pipetted into microtitre plate wells. After air drying, the wells were blocked with BSA-PBS (1\% bovine serum albumine in phosphate-buffered saline) for 1-h. PBS concentration was $\left(10 \mathrm{mM} \mathrm{PO}_{4}^{3-}, 137 \mathrm{mM} \mathrm{NaCl}\right.$, and $2.7 \mathrm{mM} \mathrm{KCl}$; $\mathrm{pH} 7,4)$. After six fold washing with PBS, $100 \mu \mathrm{l}$ of sera diluted 1:20 to 1:5000 in BSA-PBS, were added to each well and incubated overnight. After that, the plates were washed six fold thoroughly with PBS. Binding was detected following a 2 -h incubation period with BSA-PBS diluted $(1 / 3200)$ peroxidase-conjugated goat anti-human IgG antibodies (Bul Bio Ltd., NCIPD, Sofia). All the incubation steps were performed at $4^{\circ} \mathrm{C}$. After six fold washing with PBS, color development was achieved in a substrate solution containing $15 \mathrm{mM} \mathrm{O}$-phenilendiamine and $0.015 \% \mathrm{H}_{2} \mathrm{O}_{2}$ in $0.1 \mathrm{M}$ sodium acetate buffer $\left(0,2 \mathrm{M} \mathrm{CH}_{3} \mathrm{COONa} / 0,2\right.$ $\mathrm{M} \mathrm{CH}_{3} \mathrm{COOH} ; \mathrm{pH} 5.0$ ) at $20^{\circ} \mathrm{C}$. The reaction was stopped after $30 \mathrm{~min}$ by addition of $50 \mu \mathrm{l}$ of $1 \mathrm{~N} \mathrm{H}_{2} \mathrm{SO}_{4}$ and the optical density (OD) was read spectrometrically at $490 \mathrm{~nm}$ by ELISA reader (TECAN, Sunrise TM, Austria). Non-specific antibody binding (OD value in a well not containing GM1 nor GD1a, nor GM3 ganglioside) was subtracted from each measurement. Patients were considered strongly positive only if the mean OD of their sera exceeded $2 \pm$ SD (standard deviation) of the healthy controls. Determinations were carried out in triplicate.

\section{Data analysis}

Results are reported as mean values \pm SEM of three independent experiments and as relative part in \%. Statistically analyzed by Student's t-test using statistical package. Differences were regarded as significant at $\mathrm{p}<0.05$.

\section{Results and discussion}

One of the possible treatments for autoimmune diseases by means of mechanical treatment of the blood, is plasmapheresis. It is effectively used in MS patients in attack [10-12]. It is applied to such patients combined with immunosuppressive treatment to suppress the basic process. A secondary increase in the synthesis of autoantibodies after plasmapheresis, has been observed in such patients which is another 
reason for the use of immunosuppressants in plasmapheresis.

Renal diseases-related metabolic abnormalities cause diverse CNS disturbances, namely uremic encephalopathy, seizures, stroke, movement disorders, sleep alterations, and peripheral nervous system involvement comprising polyneuropathy, mononeuropathies, and myopathy. Some inherited and acquired renal diseases present with concomitant or precedent neurologic syndromes. Several mechanisms involved include toxic metabolic accumulation, hyperkalemia, hypercoagulability, immunologic disturbances, and tubular acidobasic disequilibrium. Clinical symptoms usually indicate severe renal dysfunction, but subtle abnormalities may occur. Judiciously tailored renal replacement therapy may avoid these complications, whereas others may emerge from these very therapies with overlapping clinical pictures. This makes an already complex management of renal patients even more difficult and asks for tight collaboration between nephrologists and neurologists [13].

In patients with end-stage renal disease, nervous system dysfunction remains a major cause of disability. Patients with chronic renal failure who have not yet received dialysis may have symptoms ranging from mild sensorial clouding to delirium and coma. Dialysis itself is associated with at least three distinct disorders of the central nervous system as mentioned above. Peripheral neuropathy is also a major cause of disability in uremic patients. Aluminum probably contributes to the pathogenesis of dialysis dementia. Parathyroid hormone, the levels of which are elevated in patients with renal failure, also may be a uremic neurotoxin. Biochemically, brain calcium levels are elevated in renal failure, possibly because of the action of parathyroid hormone. Studies on synaptosomes have also shown that parathyroid hormone can affect calcium transport in the brain. Intellectual dysfunction, dialysis dementia, uremic neuropathy, and the dialysis disequilibrium syndrome can be diagnosed when the characteristic clinical findings are present and other causes ofnervous systemdysfunction have been excluded [2].

Our previous results obtained by ELISA technique suggested correlation between the titer of serum antibodies against GM1, GD1a and GM3 and the occurrence of demyelination, neurodegeneration and violating the integrity of the BBB $[6,14,15]$. Additionally, we have already proven that a correlation betweenthe biomarkers of chronic remitting experimental allergic encephalomyelitis (CREAE), the animal model of MS and the biomarkers in MS patients excists [14-16]. We were interested in the level of neurodegenerative and demyelinisation changes in both the CNS andPNS in case of chronic intoxication likehaemodialysis $[17,18]$.

The idea of the authors was to test the theory that the low level of toxins in the body of MS patients (consuming purified water, absence of preservatives and others in food) is directly related to the absence ofattacks and to link that condition to patients with chronic relapsing form of MS who are on haemodialysis. In the present study significantly elevated serum IgG anti-GM1a antibodies titer was detected in patient's sera while on dialysis (Figure S1). From our studies on mothers with MS we know that after a Cesarean section (presence of toxins in the body after general anesthesia), there is always accompanying weak attack during postpartum period $[16,19]$.

Nikitin S.S. [2017, Moscow] said that "Patients with urogenital problems caused by neurological suffering, often sent to the urologist, while carefully collected from the beginning of history requires participation in the conduct the patient is a neurologist. Urological

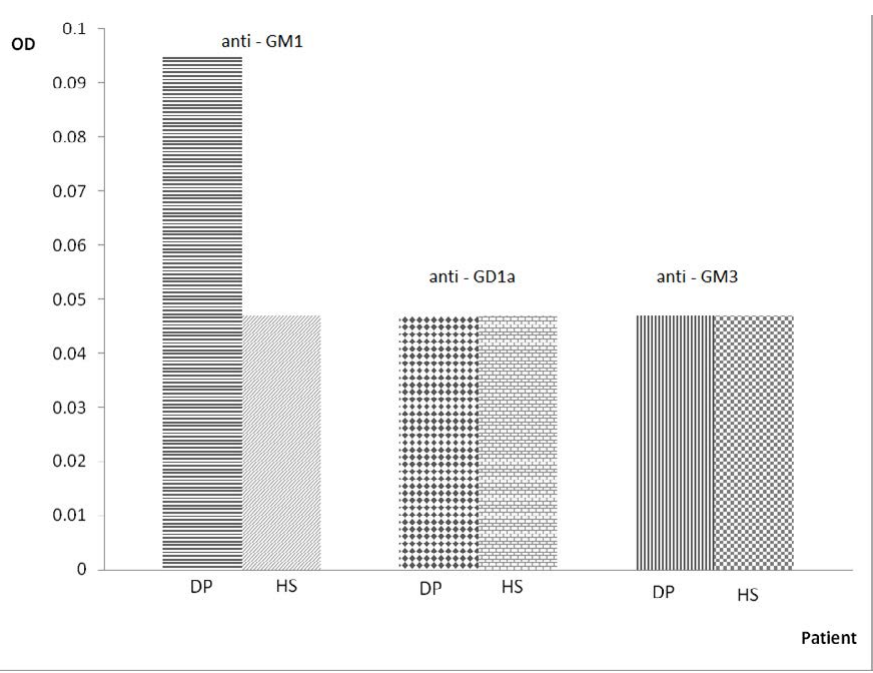

Figure 1. Optical density of the serum IgG anti-GM1, anti-GD1a and anti-GM3 antibodies in dialysis patient

OD - Optical density; DP - Dialisys patient; HS - Health subject

disorders can be early signs of hidden serious neurological disorders, as well as neurological complications deployed suffering that change the patient's lifestyle, while there are modern methods of medical treatment and support." [20].

\section{Conclusion}

Our immunological studies demonstrated that in sera of patient on dialysis there is presence of high titer of anti-GM1 antibodies. We can conclude that the observed long-term dialisys patient has a weak presence of demyelination but not the expected by us neurodegeneration, and not a case of tearing of the integrity of BBB (Figure 1). These data also suggest that there are nerve tissue changes in patients on dialysis and there is correlation between the urinary and nervous systems.

\section{Disclosures}

The authors have no disclosure to report.

\section{References}

1. Mahoney CA, Arieff AI (1982) Uremic encephalopathies: clinical, biochemical, and experimental features. Am J Kidney Dis 2: 324-336.[Crossref]

2. Fraser CL, Arieff A I (1988) Nervous system complications in uremia. Ann Intern Med 109: 143-153.

3. Lockwood AH (1989) Neurologic complications of renal disease. Neurol Clin 7: 617-627.

4. Brettschneider J, Petzold A, Junker A, Tumani H (2006) Axonal damage markers in the cerebrospinal fluid of patients with clinically isolated syndrome improve predicting conversion to definite multiple sclerosis. Mult Scler 12: 143-148.

5. Kolyovska V (2016) Serum IgG antibodies to GD1a and GM1 gangliosides in elderly people. Biomed Khim 62: 93-95.

6. Zaprianova E, Majtenyi K, Deleva D, Mikova O, Filchev A, et al. (2004) Serum IgG and IgM ganglioside GM1 antibodies in patients with multiple sclerosis. Clin Neurosci 57: 94-99.

7. Bucurescu G (2016) Neurological Manifestations of Uremic Encephalopathy Clinical Presentation. Med Scape May 17.

8. Wekerle H (2016) The gut-brain connection: triggering of brain autoimmune disease by commensal gut bacteria. Rheumatology 55 (S2): ii68-ii75.

9. Ravindranath MH, Muthugounder S (2005) Human antigangliosideautoantobodies: validation of ELISA. Ann NY Acad Sci 1050: 229-242. 
10. Tumani H (2008) Corticosteroids and plasma exchange in multiple sclerosis.J Neurol 255 Suppl 6: 36-42.

11. Matsuo H (2014) Plasmapheresis in acute phase of multiple sclerosis and neuromyelitisoptica. Nihon Rinsho 72: 1999-2002.[Crossref]

12. Jamshidian A, Gharagozloo M (2012) Canplasmaexchange therapy induce regulatory T lymphocytes in multiple sclerosis patients? Clin Exp Immunol 168: 75-77.[Crossref]

13. Lacerda G, Krummel T, Hirsch E (2010) Neurologic presentations of renal diseases. Neurol Clin 28(1): 45-59.

14. Kolyovska V, Deleva D (2012) Serum IgG and IgM antibodies to GD1a ganglioside in adults - preliminary data. ActaMorphol et Anthropol 19: 114-117.

15. Zaprianova E, Deleva D, Sultanov B, Kolyovska V (2010) Serum ganglioside GM3 changes in patients with early multiple sclerosis. Acta Morphol. etAnthropol 15: 16-18.

16. Deleva D, Kolyovska V, Sultanov B (2012) Multiple sclerosisand pregnancy: Disease biomarkers. Compt Rend Acad Bulg Sci 65: 865-870.

17. Freeman R, Lazarus M, Hickey W, Dawson DM (1982) Multiple sclerosis in association with dialysis encephalopathy syndrome. J Neurol Neurosurg Psychiatry 45: 658-659. [Crossref]

18. Fraser CL, Arieff AI (1994) Metabolic encephalopathy as a complication of renal failure: mechanisms and mediators. New Horiz 2: 518-526.

19. Klingel R, Heibges A, Fassbender C (2009) Plasmaexchange and immunoadsorption for autoimmune neurologic diseases-current guidelines and future perspectives. Atheroscler Suppl 10: 129-132.

20. Nikitin SS (2017) Neurology sevodnya 1 (01) Moscow 115478

Copyright: $@ 2017$ Kolyovska V. This is an open-access article distributed under the terms of the Creative Commons Attribution License, which permits unrestricted use, distribution, and reproduction in any medium, provided the original author and source are credited. 